\title{
Successful aging: considering non-biomedical constructs
}

This article was published in the following Dove Press journal:

Clinical Interventions in Aging

II November 2016

Number of times this article has been viewed

\author{
Lisa F Carver' \\ Diane Buchanan ${ }^{2}$ \\ 'Department of Sociology, Queen's \\ University Kingston, ON, Canada; \\ ${ }^{2}$ School of Nursing, Queen's \\ University Kingston, ON, Canada
}

\begin{abstract}
Objectives: Successful aging continues to be applied in a variety of contexts and is defined using a number of different constructs. Although previous reviews highlight the multidimensionality of successful aging, a few have focused exclusively on non-biomedical factors, as was done here.

Methods: This scoping review searched Ovid Medline database for peer-reviewed Englishlanguage articles published between 2006 and 2015, offering a model of successful aging and involving research with older adults.

Results: Seventy-two articles were reviewed. Thirty-five articles met the inclusion criteria. Common non-biomedical constructs associated with successful aging included engagement, optimism and/or positive attitude, resilience, spirituality and/or religiosity, self-efficacy and/or self-esteem, and gerotranscendence.

Discussion: Successful aging is a complex process best described using a multidimensional model. Given that the majority of elders will experience illness and/or disease during the life course, public health initiatives that promote successful aging need to employ non-biomedical constructs, facilitating the inclusion of elders living with disease and/or disability.
\end{abstract}

Keywords: successful aging, resilience, gerotranscendence, engagement, optimism

\section{Introduction}

Aging is a journey over time. Although it could be argued that we begin to age the moment we are born, generally awareness of aging is triggered by the first signs of weakness, serious illness, or the death of an elderly loved one. ${ }^{1}$ Some changes will have been easy; others painful and challenging. Given that life expectancy is increasing, ${ }^{2}$ there are a large number of people seeking to integrate their life experiences and come to terms with the inevitability of death. Understanding older adults' beliefs about successful aging can be a valuable tool for the provision of support to elders engaged in this process and for the creation of public health interventions. ${ }^{3}$ This scoping review examines the factors involved in successful aging. It did not review articles that were concerned with similar constructs, such as healthy aging or aging well, since they "struggle with similar conceptual and methodological limitations"4 without the benefit of the decades of research that exists for successful aging. Successful aging was first conceptualized as the circumstance in which an older person or elder experiences life satisfaction. ${ }^{5}$ However, research has long since abandoned life satisfaction as the criterion for successful aging and replaced it with a biomedical model, ${ }^{6}$ as shown in several reviews of the literature. For example, Depp and $\mathrm{Jeste}^{7}$ reviewed 28 articles describing large quantitative studies on successful aging published from 1978 to 2005 and discovered that $90 \%$ of the models used were at least in part biomedical. Similarly, Cosco et $\mathrm{al}^{8}$
Correspondence: Lisa F Carver Department of Sociology, D4I0 Mackintosh-Corry, Queen's University Kingston, ON K7L 3N6, Canada

Email 4lfc@queensu.ca (c)
hereby accept the Terms. Non-commercial uses of the work are permitted without any further permission from Dove Medical Press Limited, provided the work is properly attributed. For permission for commercial use of this work, please see paragraphs 4.2 and 5 of our Terms (hitps://wwww.dovepress.comm/terms.php). 
reviewed 84 articles published between 1979 and 2011, in which $92 \%$ included biomedical factors. Non-biomedical constructs found in these reviews included positive psychological traits such as resilience, optimism, sense of purpose, positive attitude toward aging, ${ }^{7}$ engagement, life satisfaction, and external factors, such as finances. ${ }^{8}$ Depression was negatively correlated with successful aging. ${ }^{9}$

In the biomedical model, successful aging requires that the elder is disease free, disease-related disability free and engaged in activities with family and/or community. ${ }^{6,10}$ This biomedical model of successful aging is discriminatory, excluding the majority of elders. For example, in one study, only $11 \%$ of Americans were aging successfully using biomedical criteria. ${ }^{11}$ Similar results were found in a European research where $8.5 \%$ of Europeans were successfully aging. ${ }^{12}$ Why does the presence of illness preclude successful aging? ${ }^{13-15}$ Many elders living with illness perceive themselves as aging successfully. ${ }^{16-18}$

Is it really efficacious to assess successful aging using a biomedical model and create policies around the results if they only apply to a small portion of those aging and exclude the majority of the population? The goal of this article was to review the non-biomedical constructs used in recent successful aging qualitative and quantitative research and to identify non-biological constructs that could be used in a model of successful aging that would include all elders, even those living with illness.

\section{Methods}

\section{Search strategy}

A scoping review ${ }^{19}$ of recent successful aging literature published between 2006 and 2015 was performed. Scoping studies "aim to map rapidly the key concepts underpinning a research area and the main sources and types of evidence available". ${ }^{8}$ Ovid Medline database was used for the search. The MEDLINE(R) database includes research from $\sim 4,600$ international journals in fields such as medicine, nursing, dentistry, veterinary medicine, allied health, and pre-clinical sciences from 1950 to the present. Since we were interested in articles written about successful aging that included a specific model, the search terms used were "successful aging" AND "model". The search was restricted to peer-reviewed English-language articles that offered a model or, at minimum, an operational definition of successful aging. To be included, the articles had to involve research with adults 40 years and older. Potentially relevant articles from the reference lists of identified publications were used if they met the inclusion criteria. All titles and abstracts from the search strategy were reviewed for eligibility. Reference lists were also examined for relevant articles. Components of successful aging were identified and categorized.

\section{Inclusion criteria}

The participants were human beings and older adults, the article was published in the English language between 2006 and 2015, successful aging was the central topic of the article, and a model or operational definition was provided.

\section{Exclusion criteria}

Articles that discussed successful aging exclusively with regard to a physiological process (eg, allostatic load), phenotypes and/or genotypes, a particular disease (eg, atherosclerosis), and a single component (eg, good cognition, diet, obesity or physical exercise) were excluded. Articles were also excluded if, despite having the term successful aging in the title, there was no discussion of successful aging in the body of the article or no definition of successful aging.

\section{Results}

Seventy-two articles resulted from this search. Abstracts were reviewed, and 35 articles met the inclusion criteria. Thirty-seven articles were excluded according to the exclusion criteria; the subject matter of excluded articles is shown in Figure 1.

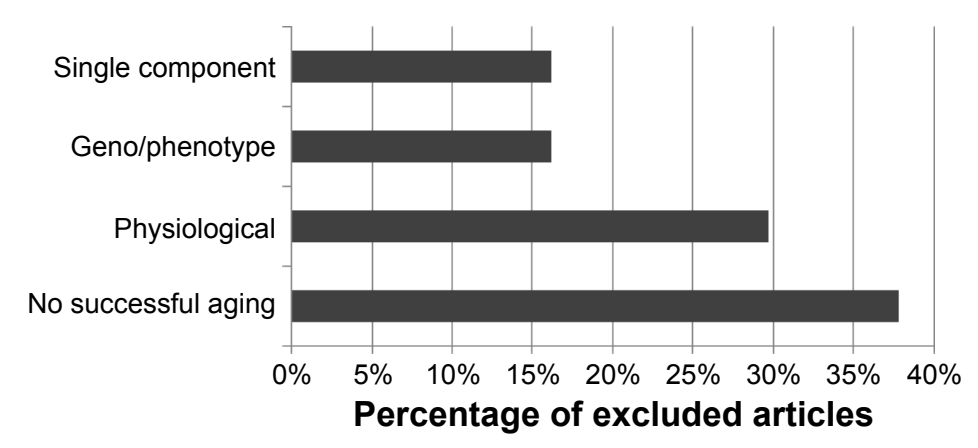

Figure I Subject of excluded articles. 
Fifty-four percent of the retained articles were quantitative studies, 37\% were qualitative studies, and 9\% were mixed methods design. The majority of articles (63\%) involved participants aged 60 years or older. Twenty-nine percent of articles included participants aged 50 years or older. Six percent of studies included participants older than 70 years. One article included participants who were 40 years and older. The oldest participants included were 90-100 years old.

All the articles reviewed employed multidimensional models of successful aging. For the majority of the articles reviewed $(71 \%)$, successful aging was synonymous with being physically healthy or disability free. Other common biomedical components employed to describe successful aging were mental health (43\%) and cognitive function $(32 \%)$. Thirty-seven percent of the included articles employed a version of the biomedical MacArthur model created by Rowe and Kahn ${ }^{6,10}$ which includes: 1) a low probability of disease and disease-related disability; 2) high cognitive and physical functional capacity (no problems with activities of daily living); and 3) active engagement with life (measured by an individual's interpersonal relations and participation in productive activity, not including time spent in solitary activity). The majority of these articles added non-biomedical constructs to Rowe and Kahn's biomedical model.

Non-biomedical constructs used in the included articles are reviewed and shown in Figure 2. Similar constructs, although not identical, are discussed together under a single heading (eg, social engagement, social support, and social contact are all discussed under the heading "engagement"). Some of the articles included constructs that could also be considered outcome measures (eg, self-rated successful aging, well-being, life satisfaction) in their successful aging models. These constructs were not categorized as non-biomedical constructs here. Health protective behaviors (eg, non-smoking, frequent exercise) were also excluded from this discussion.

\section{Non-biomedical constructs of successful aging}

Non-biomedical constructs associated with successful aging included engagement, ${ }^{12,20-32}$ optimism and/or positive attitude, ${ }^{33-36}$ resilience (including coping), ${ }^{37-40}$ self-efficacy and/or self-esteem, ${ }^{41-43}$ and gerotranscendence. ${ }^{44,45}$ Spirituality and/or religiosity were included as an important factor in more than one-third of articles, ${ }^{14,26,43,46-49}$ but not necessarily in the models of successful aging.

\section{Engagement}

Engagement was the most frequently used construct. Sixty-three percent of articles reviewed included engagement in their successful aging models (Figure 2). In the articles reviewed, engagement was expressed as active engagement, ${ }^{20-22}$ caring engagement $(\mathrm{CE})$ and productive engagement (PE), ${ }^{23}$ and social activity dimensions. ${ }^{12,31}$

Active engagement was defined as including $<35$ hours per week in solitary activities (eg, video gaming, reading), community membership, and volunteering. ${ }^{20}$ In the study by Weir et al, ${ }^{20}$ the majority (83\%) of the 14,749 participants, elders older than 60 years, had one or more chronic conditions and therefore could not be classified as successful agers within the constraints of the biomedical model. However, 69\% were actively engaged in life. Using a bivariate logistic regression analysis, they found that increased age was significantly related to engagement with life. In fact, participants who were between 70 and 74 years were more engaged than those between 60 and 64 years (odds ratio 0.92, $95 \%$ confidence interval $0.916-0.933$ ). Weir et al $^{20}$ suggest that it is important to note the changes in engagement over the lifespan.

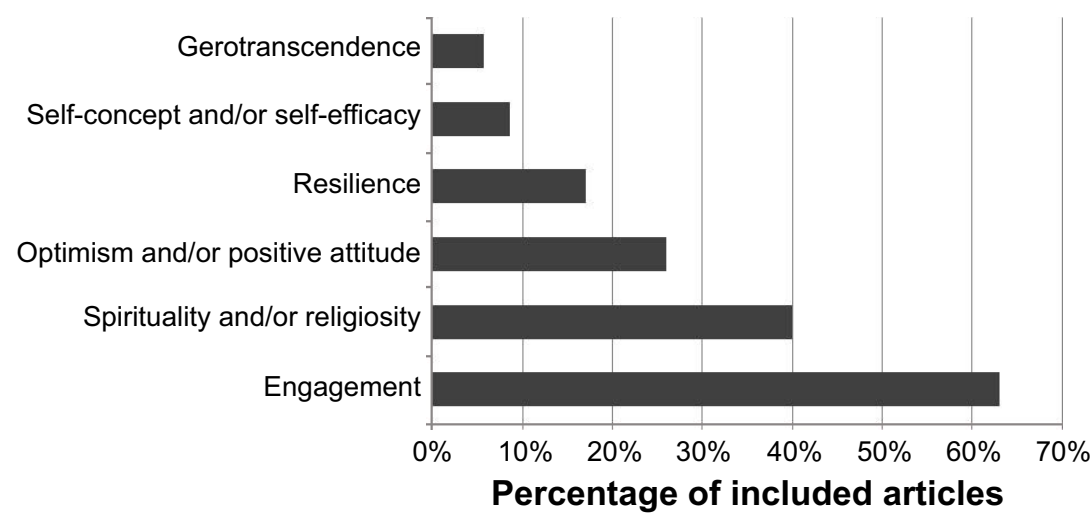

Figure 2 Components of successful aging articles included in scoping review. 
$\mathrm{CE}$ and $\mathrm{PE}$ is defined as support given to significant others (eg, friends, family), and PE is represented by activities that contributed to others (individuals such as family, organizations such as workplace or the community). In one study, results among 2,120 participants indicated that the means on CE $(\eta=0.383, P<0.001)$ and PE $(\eta=0.343, P<0.001)$ were predictive of successful and unsuccessful aging. ${ }^{23}$

Social activity was the third conceptualization of engagement used to explain successful aging. The social activity/ engagement dimension was defined as involving participation in volunteer work and/or care in the form of babysitting grandchildren or interaction in the last month with family, spouse, or community. ${ }^{12}$ Using data combined from 14 European countries and Israel, Hank ${ }^{12}$ used a logistic model to examine successful aging and found a weak relationship between engaged and physically healthy elders $\left(R^{2}=0.05\right)$. Overall, using the biomedical criteria, successful aging was found in only $8.5 \%$ of the 21,493 participants. Hank ${ }^{12}$ concluded that there was a lack of engagement among a large number of older adults with good health.

Community engagement is another component of successful aging. Qualitative studies among Alaska Natives found that successful aging was defined by participants as achieving a respected role in one's community. ${ }^{22}$ Unsuccessful aging was defined as low activity, alcohol consumption, and loss of faith in oneself. ${ }^{21}$ Younger participants believed that their current physical health was a predictor of the ability to age successfully, whereas elders linked successful aging to the ability to maintain mental health and participate within their community. ${ }^{21}$

In the majority of the reviewed articles, engagement seemed to have a significant association with age, ${ }^{20}$ and in some cases, could be used to discriminate between those aging successfully and those who were not. ${ }^{21-23}$ Some participants indicated that engagement was the expression of a decision to maintain involvement in activities and with others, despite the challenges of health crises. ${ }^{29}$ In one study, engagement and physical health were weakly correlated, ${ }^{12}$ suggesting that physical health may not be a good predictor of successful aging within a nonbiomedical model.

\section{Optimism and/or positive attitude}

Optimism and positive attitude are similar psychological characteristics that involve the expectation that events will resolve with the best possible outcome..$^{50}$ In $26 \%$ of the articles, optimism and/or positive attitude were proposed as important constructs in the understanding of successful aging.
Optimism and positive attitude have been associated with life satisfaction and have similar effects on individuals irrespective of socioeconomic status or physical health. ${ }^{51}$ Optimism and positive attitude can be integral in the transition from poor health to better, possibly because they are believed to support resilience. ${ }^{36}$ The presence of optimism and positive attitude is associated with the ability to manage later life adversity ${ }^{36}$ and is integral to the experience of successful aging. ${ }^{14}$

\section{Resilience}

A number of researchers have proposed that successful aging requires psychological resources to enable individuals to adapt in the face of the challenges created by illness, loss of loved ones, and changes in functional ability. ${ }^{39,40,52}$ Resilience is a psychological resource that facilitates adaptation and is associated with characteristics of successful aging. ${ }^{39,40}$ As shown in Figure 2, 17\% of the articles included here employed resilience as a component of their successful aging model.

Resilience was significantly correlated with the components of successful aging, including well-being ( $r=0.494$, $P<0.001)$, optimism $(r=0.438, P<0.001)$, self-rated successful aging ( $r=0.425, P<0.001)$, and to a lesser extent, physical function $(r=0.116, P<0.001) .{ }^{39}$ In a sample of older adults, many of whom were living with illness, older age was associated with higher self-rated health. ${ }^{52}$ In this research, resilience, depression, and physical health had similar impacts on successful aging. ${ }^{52}$

Resilience is not just a psychological trait; it can also be considered as a process. Resilience as a process is a compensatory response to adversity or risk. Aging brings many forms of adversity, including potential loss of loved ones, illness, and decline in functional abilities. In this study, ${ }^{40}$ qualitative interviews with two individuals were used to explore resilience and successful aging among older adults living with dementia, a major source of adversity. The results indicated that resilience can be present among people living with dementia and that they can live meaningful lives. Harris ${ }^{40}$ suggests that there is an:

'Ordinary magic' of human beings present within us, regardless of age, the resilience of the human spirit, the human capacity to adapt and survive in the face of adversity

Rather than considering successful aging as a consequence of perfect health, it could be considered a reflection, at least in part, of resilience in the face of the adversity that life presents - a far more obtainable goal for most older adults. 
In the reviewed articles, successful agers exhibited resilience in the presence of physical and mental health challenges. ${ }^{53}$ Higher resilience among those living with illness was associated with successful aging levels similar to those of healthy adults. ${ }^{52}$ It must be noted that resilience in older adults does not apply to life in a unified manner and may be manifested in some areas of life and not others. ${ }^{40}$ These associations between resilience and successful aging suggest that it is a valuable predictive construct and useful to be included in successful aging models. ${ }^{39,52}$

\section{Spirituality and/or religiosity}

In this context, spirituality can include, but is not limited to, religious activities and experiences such as serenity, appreciation of life, and altruistic activities; ${ }^{14}$ encompassing meaningful interactions with a supreme being, art, culture, nature, family, and/or animals. Spirituality is not constrained by institutions or formal rituals. Religiosity is "used to describe a formal system of worship that is based on social interaction with others who share similar beliefs". ${ }^{54}$ As a broad construct, spirituality and/or religiosity and/or religion were hypothesized to be important aspects of successful aging in $40 \%$ of the articles, but only a few researchers included them in their successful aging model. ${ }^{14,15}$

Spirituality, defined as including religious/spiritual activity and motivation, ${ }^{15}$ was significantly correlated with demographic variables, including education, income, marital status, and ethnicity. Spirituality was also significantly correlated with the Connor-Davidson Scale for Resilience score ${ }^{55}(\rho=0.282$, $P<0.001)$, Life Events Scale score $(\rho=0.118, P<0.001)$, and Life Orientation Test score $(\rho=0.106, P<0.001) .{ }^{15}$ Spirituality was not significantly correlated with age $(\rho=0.005, P=0.840)$, depression $(\rho=-0.018, P=0.4768)$, physical health $(\rho=0.036$, $P=0.128)$, mental health $(\rho=0.042, P=0.071)$, or self-rated successful aging $(\rho=0.064, P=0.499) .{ }^{15}$

Religion can be considered intrinsic (eg, religion is for personal comfort) and extrinsic (eg, religion provides membership in a social group).$^{51}$ Extrinsic religiosity significantly correlated with well-being $(r=-0.34, P<0.01)$, health $(r=-0.31, P<0.05)$, and education $(r=-0.27$, $P<0.05) .{ }^{54}$ Intrinsic religiosity significantly correlated with age $(r=0.28, P<0.05)$, education $(r=-0.28, P<0.05)$, and internal religiosity $(r=0.57, P<0.01) .{ }^{54}$

Weak associations have been found, in some cases, between spirituality and/or religiosity and hypothesized components of successful aging, ${ }^{43,48,54}$ suggesting that although spirituality and/or religiosity may be important for some elders, ${ }^{47}$ this construct is not necessarily predictive of successful aging when included in a model.

\section{Self-efficacy and/or self-esteem}

Self-esteem, self-efficacy, interpersonal relationships, and self-achievement have been shown to contribute to a significant predictive model $(F=159.09, P<0.001)$, explaining $69 \%$ of the variance in successful aging. ${ }^{51}$ Self-esteem had the greatest influence in the model $(\beta=0.38$, standard error $(\mathrm{SE})=0.06, P<0.001)$, followed by self-achievement ( $\beta=0.25, \mathrm{SE}=0.07, P<0.001)$, interpersonal relationships $(\beta=0.22, \mathrm{SE}=0.07, P<0.001)$, and self-efficacy $(\beta=0.21$, $\mathrm{SE}=0.08, P<0.001) .{ }^{56}$ Successful aging was also related to self-acceptance, self-contentment, engagement, and selfgrowth. ${ }^{41}$

Self-efficacy, self-esteem, and/or self-acceptance appear to have value as predictors of successful aging. ${ }^{41-43,57}$ However, more research is required to better understand the role of different aspects of self-efficacy, such as self-esteem, in successful aging.

\section{Gerotranscendence}

Gerotranscendence involves a shift in perspective and the recognition that the task at hand is self-awareness and preparation for death. ${ }^{58,59}$ The elders may desire an increase in close relationships ${ }^{45}$ or prefer to spend more time in solitude. Gerotranscendence includes an acceptance of life as meaningful, purposeful, and coherent, as well as feelings of connection to earlier generations. ${ }^{60}$ It may also involve the acceptance of religion or just peace of mind. ${ }^{59}$

Gerotranscendence is the ability to be positive as life is ending $^{61}$ and to take time for retrospection. Engagement in career has generally ended and the past is revisited. ${ }^{58}$ The goal is the attainment of wisdom and pursuit of meaning, and elders' lived experiences reflect this. ${ }^{41}$ This notion of a resilient self in older age, ${ }^{62}$ which develops wisdom through gerotranscendence, was proposed by Erikson ${ }^{63}$ and confirmed in later research. ${ }^{44,59}$

Associations between gerotranscendence and outcome measures frequently associated with successful aging were confirmed in significant correlations between the Successful Aging Inventory and the measures of life satisfaction $(r=0.263, P<0.01)$ and purpose in life $(r=0.361, P<0.000) .{ }^{44}$ This relationship was also confirmed in a Japanese study when gerotranscendence was significantly correlated with life satisfaction (coherence subscale $r=0.40, P<0.001$; solitude subscale $r=-0.32, P<0.001) .{ }^{60}$ The negative correlation between the solitude subscale and life satisfaction 
may reflect the rejection of social interaction, a component of life satisfaction. ${ }^{60}$

Gerotranscendence includes, but is not limited to, aspects of spirituality and has shown some predictive ability in successful aging research. ${ }^{64}$ It is a promising construct that needs to be further explored in the context of successful aging.

\section{Discussion}

The multidimensionality of successful aging is now acknowledged in the literature. ${ }^{28,39}$ However, little agreement exists on the relevant dimensions of successful aging. The goal of this article was to review recent literature on successful aging and identify the non-biomedical constructs most frequently employed. Like other reviews of successful aging constructs, ${ }^{8,9}$ a majority of the articles included similar biomedical constructs in their models but were heterogeneous in terms of their non-biomedical constructs. Non-biomedical constructs of successful aging found in this review included engagement, ${ }^{12,20,23}$ optimism and/or positive attitude, ${ }^{33,35,36}$ resilience, ${ }^{38-40}$ spirituality and/or religiosity, ${ }^{14,15}$ self-efficacy and/or self-esteem, ${ }^{41-43}$ and gerotranscendence. ${ }^{41,44}$

The biomedical model, employed by the majority of articles reviewed, discriminates against elders who feel that they are aging successfully with disease and/or disability. ${ }^{20,33,65,66}$ The biomedical model also perpetuates the myth that illness/ physical health results in unsuccessful aging. ${ }^{6,10,66}$ In order to reframe successful aging as an inclusive process, the models need to include concepts outside biomedical criteria such as the non-biomedical constructs found in this review: engagement, optimism and/or positive attitude, resilience, spirituality and/or religiosity, self-efficacy and/or self-esteem, and gerotranscendence.

\section{Limitations}

The limitations of this review included the restricted search criteria. Examining the various models built around specific perspectives (eg, feminist) or disease-related groups were beyond the scope of this article as was the inclusion of the terms healthy aging or aging well. The number of articles found would have been greater if we had included these perspectives and related terms such as aging well. It is also important to recognize that the choice of both successful aging model and outcome measures profoundly impacts the results. ${ }^{12}$ Another limitation is the lack of consistency in the operational definitions of these constructs (eg, engagement), which results in an inability to compare the study results accurately. Measurement tools selected in each research project reflected the models proposed within, and therefore, it is difficult to compare results from one study to another because each includes and measures different constructs.

\section{Clinical implications}

Over the last several decades, there has been a move to consider the whole patient when designing treatment protocols. This scoping review suggests that by stepping away from the definition of success in aging as non-diseased and embracing the idea that people with illness can continue to self-define as successful agers, we can build a clinician-patient interface that acknowledges the patient as a person who happens to have an illness. Knowing that patients/clients are aging successfully with illness may relieve some pressure on clinicians in situations when a cure is impossible. In the context of chronic disease, it may be appropriate for clinicians to focus on the quality of life, in terms of both pharmaceutical and behavioral prescriptions. It may be possible, by inquiring about patient engagement with his/her social network, optimism or positive attitude, resilience, spirituality, elements of gerotranscendence, and self-esteem and self-efficacy that the clinician can ascertain the potential for successful aging with a particular diagnosis. Furthermore, many of these constructs (eg, resilience, self-esteem) can be fostered through education. Successful aging could be bolstered by suggesting programs to build skills in these areas along with the usual treatment modalities.

\section{Conclusion}

This is not the first article to call for modifications to the biomedically based MacArthur model of successful aging. In fact, according to a recent article by Rowe and Kahn, ${ }^{66}$ at least 100 variations to this model have been suggested. The unique element of this study is that we compiled the non-biomedical constructs used in the recent research. As suggested in another recent article:

The task ahead is to agree on key concepts and definitions, understand plausible pathways, and develop comprehensive multisector and intersectoral approaches to support healthy ageing and in particular older adults. ${ }^{67}$

Our scoping review provides contemporary constructs that can be used in successful aging model building in the hopes of encouraging future successful aging research that is inclusive of those aging with illness. This list of non-biomedical constructs can also be utilized by clinicians to help assess the internal resources that clients/patients have and identify the skills and services they may need in order to age successfully with a diagnosis of illness.

The right to participate as active members of society and community and to engage in self-determination are vital 
components of meaningful life for all elders, regardless of physical health and (dis)ability. ${ }^{68,69}$ Clinical initiatives and public health programs that seek to increase successful aging, including enhancing elders' engagement in life and community, must be inclusive of all elders, not just the healthy ones. In the clinical context, we need to consider that non-biomedical components of successful aging are often modifiable and engage in referrals to public programs designed to enhance these modifiable characteristics. By developing skills related to constructs that are predictive of successful aging, there is the potential for improvement in the quality of life and possibly better health outcomes for a broad population of elders.

\section{Disclosure}

The authors report no conflicts of interest in this work.

\section{References}

1. Shin KR, Kim MY, Kim YH. Study on the lived experience of aging. Nurs Health Sci. 2003;5(4):245-252.

2. Cho J, Martin P, Poon LW. The older they are, the less successful they become? Findings from the Georgia Centenarian Study. J Aging Res. 2012;2012:1-8.

3. Phelan EA, Anderson LA, LaCroix AZ, Larson EB. Older adults' views of "successful aging" - how do they compare with researchers' definitions? J Am Geriatr Soc. 2004;52(2):211-216.

4. Pruchno RA. Successful aging: contentious past, productive future. Gerontologist. 2015;55(1):1-4.

5. Havighurst RJ. Successful aging. Gerontologist. 1961;1:8-13

6. Rowe JW, Kahn RL. Successful aging. Gerontologist. 1997;37(4): 433-440.

7. Depp CA, Jeste DV. Definitions and predictors of successful aging: a comprehensive review of larger quantitative studies. Am J Geriatr Psychiatry. 2006;14(1):6-20.

8. Cosco TD, Prina AM, Perales J, Blossom CM, Brayne S, Brayne C. Operational definitions of successful aging: a systematic review. Int Psychogeriatr. 2014;26(3):373-381.

9. Jeste DV, Depp CA, Vahia IV. Successful cognitive and emotional aging. World Psychiatry. 2010;9(2):78-84.

10. Rowe JW, Kahn RL. Successful Aging. New York, NY: Pantheon Books; 1998

11. McLaughlin SJ, Connell CM, Heeringa SG, Li LW, Roberts JS. Successful aging in the United States: prevalence estimates from a national sample of older adults. J Gerontol B Psychol Sci Soc Sci. 2010; 65B(2):216-226

12. Hank K. How "Successful" do older Europeans age? Findings from SHARE. J Gerontol B Psychol Sci Soc Sci. 2011;66B(2):230-236.

13. Donnellan C, Hevey D, Hickey A, O'Neill D. Adaptation to stroke using a model of successful aging. Aging Neuropsychol Cogn. 2012;19(4): 530-547.

14. Iwamasa GY, Iwasaki MA. New multidimensional model of successful aging: perceptions of Japanese American older adults. J Cross Cult Gerontol. 2011;26(3):261-278.

15. Vahia IV, Depp CA, Palmer BW, et al. Correlates of spirituality in older women. Aging Ment Health. 2011;15(1):97-102.

16. Knight T, Ricciardelli LA. Successful aging: perceptions of adults aged between 70 and 101 years. Int J Aging Hum Dev. 2003;56(3): 223-245.

17. Strawbridge WJ, Wallhagen MI, Cohen RD. Successful aging and well-being: self-rated compared with Rowe and Kahn. Gerontologist. 2002;42(6):727-733
18. Montross LP, Depp C, Daly J, et al. Correlates of self-rated successful aging among community-dwelling older adults. Am J Geriatr Psychiatry. 2006;14(1):43-51.

19. Arksey H, O’Malley L. Scoping studies: towards a methodological framework. Int J Soc Res Methodol. 2005;8(1):19-32.

20. Weir PL, Meisner BA, Baker J. Successful aging across the years: does one model fit everyone? J Health Psychol. 2010;15(5):680-687.

21. Lewis JP. Successful aging through the eyes of Alaska Native elders: exploring generational differences among Alaska Natives. J Cross Cult Gerontol. 2010;25(4):385-396.

22. Lewis JP. Successful aging through the eyes of Alaska Native elders. What it means to be an elder in Bristol Bay, AK. Gerontologist. 2011; 51(4):540-549.

23. Ng SK, Cheung C-K, Chong AML, Woo J, Kwan AYH, Lai S. Aging well socially through engagement with life: adapting Rowe and Kahn's model of successful aging to Chinese cultural context. Int J Aging Hum Dev. 2011;73(4):313-330.

24. Parslow RA, Lewis VJ, Nay R. Successful aging: development and testing of a multidimensional model using data from a large sample of older Australians. J Am Geriatr Soc. 2011;59(11):2077-2083.

25. Liu JL, Richardson PK. Successful aging in older adults with disability. OTJR. 2012;32(4):126-134.

26. Troutman-Jordan M, Nies MA, Davis B. An examination of successful aging among Southern Black and White older adults. J Gerontol Nurs. 2013;39(3):42-52.

27. Cohen CI, Pathak R, Ramirez PM, Vahia I. Outcome among community dwelling older adults with Schizophrenia: results using five conceptual models. Community Ment Health J. 2009;45(2):151-156.

28. Young Y, Frick KD, Phelan EA. Can successful aging and chronic illness coexist in the same individual? A multidimensional concept of successful aging. J Am Med Dir Assoc. 2009;10(2):87-92.

29. Rossen EK, Knafl KA, Flood M. Older women's perceptions of successful aging. Act Adapt Aging. 2008;32(2):73-88.

30. Duay DL, Bryan VC. Senior adults' perceptions of successful aging. Educ Gerontol. 2006;32(6):423-445.

31. Flood M, Scharer K. Creativity enhancement: possibilities for successful aging. Issues Ment Health Nurs. 2006;27(9):939-959.

32. Hsu HC. Gender disparity of successful aging in Taiwan. Women Health. 2005;42(1):1-21.

33. Bowling A. Lay perceptions of successful ageing: findings from a national survey of middle aged and older adults in Britain. Eur J Ageing. 2006;3(3):123-136.

34. Torres S, Hammarström G. Successful aging as an oxymoron: older people with and without home-help care talk about what aging well means to them. IJAL. 2009;4(1):23-54

35. Bowling A, Iliffe S. Psychological approach to successful ageing predicts future quality of life in older adults. Health Qual Life Outcomes. 2011;9(13):1-10.

36. Van Wagenen A, Driskell J, Bradford J. "I'm still raring to go": successful aging among lesbian, gay, bisexual and transgender older adults. J Aging Stud. 2013;27(1):1-14.

37. Wagnild G. Resilience and successful aging: comparison among low and high income older adults. J Gerontol Nurs. 2003;29(12): 42-49.

38. Kahana E, Kelley-Moore J, Kahana B. Proactive aging: a longitudinal study of stress, resources, agency, and well-being. Aging Ment Health . 2012;16(4):438-451.

39. Lamond AJ, Depp CA, Allison M, et al. Measurement and predictors of resilience among community dwelling older women. J Psychiatr Res. 2009;43(2):148-154.

40. Harris PB. Another wrinkle in the debate about successful aging: the undervalued concept of resilience and the lived experience of dementia. Int J Aging Hum Dev. 2008;67(1):43-61.

41. Reichstadt J, Sengupta G, Depp C, Palinkas LA, Jeste DV. Older adults' perspectives on successful aging: qualitative interviews. Am J Geriatr Psychiatry. 2010;18(7):567-575.

42. Cha H, Seo EJ, Sok SR. Factors influencing the successful aging of older Korean adults. Contemp Nurse. 2012;41(1):78-87. 
43. Vahia IV, Thompson WK, Depp CA, Allison M, Jeste DV. Developing a dimensional model for successful cognitive and emotional aging. Int Psychogeriatr. 2012;24(4):515-523.

44. Troutman M, Nies MA, Small S, Bates A. The development and testing of an instrument to measure successful aging. Res Gerontol Nurs. 2011;4(3):221-232.

45. Troutman-Jordan M, Staples J. Successful aging from the viewpoint of older adults. Res Theory Nurs Pract. 2014;28(1):87-104.

46. Liu H, Byles JE, Xu X, Zhang M, Wu X, Hall JJ. Evaluation of successful aging among older people in China: results from China health and retirement longitudinal study. Geriatr Gerontol Int. Epub 2016 Aug 10.

47. Pruchno RA, Wilson-Genderson M, Rose M, Cartwright F. A two-factor model of successful aging. Gerontologist. 2010;50(6):821-833.

48. Vance DE, Brennan M, Enah C, Smith GL, Kaur J. Religion, spirituality, and older adults with HIV: critical personal and social resources for an aging epidemic. Clin Interv Aging. 2011;6:101-109.

49. Nagalingam J. Understanding successful aging: a study of older Indian adults in Singapore. Care Manag J. 2007;8(1):18-25.

50. Carver CS, Scheier MF, Segerstrom SC. Optimism. Clin Psychol Rev. 2010;30(7):879-889.

51. Alarcon GM, Bowling NA, Khazon S. Great expectations: a metaanalytic examination of optimism and hope. Pers Individ Dif. 2013; 54(7):821-827.

52. Jeste DV, Savla GN, Thompson WK, et al. Association between older age and more successful aging: critical role of resilience and depression. Am J Psychiatry. 2013;170(2):188-196.

53. Moore RC, Eyler LT, Mausbach BT, et al. Complex interplay between health and successful aging: role of perceived stress, resilience, and social support. Am J Geriatr Psychiatry. 2015;23(6):622-632.

54. Hilton JM, Child SL. Spirituality and the successful aging of older Latinos. Couns Values. 2014;59:17-34.

55. Connor K, Davidson JRT. Development of a new resilience scale: the Connor-Davidson Resilience Scale (CDRISC). J Depress Anxiety. 2003;18(2):76-82.

56. Coyne JC, Tennen H, Ranchor AV. Positive psychology in cancer care: a story line resistant to evidence. Ann Behav Med. 2010;39(1):35-42.
57. Araújo L, Ribeiro O, Teixeira L, Paúl C. Successful aging at 100 years: the relevance of subjectivity and psychological resources. Int Psychogeriatr. 2016;28(2):179-188.

58. Erikson JM. The Lifecycle Completed: Erik H. Erikson. New York, NY: W.W. Norton; 1997.

59. Tornstam L. Gerotranscendence: A Developmental Theory of Positive Aging. New York, NY: Springer; 2005.

60. Hoshino K, Zarit SH, Nakayama M. Development of the gerotranscendence scale type 2: Japanese version. Int J Aging Hum Dev. 2012; 75(3):217-237.

61. Nystrom A, Andersson-Segesten K. Peace of mind as an important aspect of old people's health. Scand J Caring Sci. 1990;4(2):55-62.

62. Baltes PB, Baltes MM. Successful Aging: Perspectives from the Behavioral Sciences. New York, NY: Cambridge University Press; 1990.

63. Erikson EH. Childhood and Society. New York, NY: W.W. Norton; 1963.

64. Melin-Johansson C, Eriksson U, Segerback I, Bostrom S. Reflections of older people living in nursing homes. Nurs Older People. 2014; 26(1):33-39.

65. Dillaway HE, Byrnes M. Reconsidering successful aging. A call for renewed and expanded academic critiques and conceptualizations. J Appl Gerontol. 2009;28:702-722.

66. Rowe JW, Kahn RL. Kahn successful aging 2.0: conceptual expansions for the 21st century. J Gerontol B Psychol Sci Soc Sci. 2015;70(4): 593-596.

67. Sadana R, Blas E, Budhuwani S, Koller T, Paraje G. Healthy ageing: raising awareness of inequalities, determinants, and what could be done to improve health equity. Gerontologist. 2016;56(suppl 2):S178-S193.

68. Ontario Human Rights Commission. Time for Action. Advancing Human Rights for Older Ontarians; 2001. Available from: http://www.ohrc. on.ca/en/time-action-advancing-human-rights-older-ontarians. Accessed October 17, 2016.

69. United Nations General Assembly Resolution 46/91. Implementation of the International Plan of Action on Ageing and Related Activities. U.N. GAOR, 46th Sess., 74th plen.mtg., Annex 1 paras.1-18, U.N. Doc.A/RES/46/91. New York: United Nations; 1991.
Clinical Interventions in Aging

\section{Publish your work in this journal}

Clinical Interventions in Aging is an international, peer-reviewed journal focusing on evidence-based reports on the value or lack thereof of treatments intended to prevent or delay the onset of maladaptive correlates of aging in human beings. This journal is indexed on PubMed Central, MedLine,

\section{Dovepress}

CAS, Scopus and the Elsevier Bibliographic databases. The manuscript management system is completely online and includes a very quick and fair peer-review system, which is all easy to use. Visit http://www.dovepress. com/testimonials.php to read real quotes from published authors. 